\title{
APLICAÇÃO DE WAVELETS NA ANALISE DE RÁDIO EMISSÕES SOLARES
}

\section{APPLICATION OF WAVELETS IN SOLAR RADIO EMISSIONS DATA}

\author{
Humberto Gimenes Macedo ${ }^{1}$ \\ Zuleika Auxiliadora da Luz Sodré \\ Francisco Carlos Rocha Fernandes ${ }^{3}$
}

Resumo: O estudo de sinais não estacionários necessita de modelos matemáticos mais sofisticados para sua análise, pois, em tais sinais, a propriedade não se mantém constante, necessitando a determinação da ocorrência no tempo de propriedades associadas à natureza do evento, como periodicidades e frequências. Nesse cenário, a análise de séries temporais por wavelet se apresenta com um valioso método matemático, pois concebe uma representação tempo-frequência, permitindo determinar quando as componentes de frequência ocorrem no tempo. Neste trabalho, uma rotina desenvolvida em MATLAB para análise por wavelet foi aplicada em dados de rádio emissões solares tipo I (02/08/2010 11:00:31 11:15:31 UT) e tipo III (09/05/2015 16:29:38 - 16:45:00 UT) registradas pelo espectrógrafo BLEN7M da rede e-CALLISTO, com o intuito de revelar periodicidades e intermitências associadas aos eventos analisados. De forma preliminar, a análise por wavelet identificou periodicidades entre 32 e 64 segundos entre a ocorrência dos grupos de emissões tipo III e de cerca de 64 segundos e de cerca de 64 segundos para correntes de emissões tipo I, demonstrando a eficácia da rotina na detecção de periodicidades nos dados espectrais solares.

Palavras-chave: Wavelet; séries temporais; rádio emissão solar tipo I; rádio emissão solar tipo III.

Abstract: The study of non-stationary signals requires more sophisticated mathematical models for analysis, for in such signs, the property does not remain constant, requiring determination of occurrence in time of properties associated with the nature of the event, as periodicities and frequencies. In this scenario, the wavelet analysis is presented as a valuable mathematical method; it conceives a timefrequency representation, allowing determining when frequency components occur in time. In this work, a routine developed in MATLAB for time series analysis by wavelet was applied to type I solar radio emissions (02/Aug/2010 11:00:31 to 11:15:31 UT) and type III (05/Sept/2015 16:29:38 to 16:45:00 UT) recorded by the spectrograph BLEN7M from e-CALLISTO network, in order to reveal periodicities and flashes associated with the events analyzed. Preliminarily, the analysis wavelet identified periodicities between 32 and 64 seconds between the occurrence of the groups of type III emissions and about 64 seconds for ichains of type I emissions, within a group, demonstrating the effectiveness of the routine in periodicity detection spectral data solar.

Keywords: Wavelet; time series; solar radio emission type I; solar radio emission type III.

\footnotetext{
${ }^{1}$ Graduando em Engenharia da Computação - Universidade do Vale do Paraíba - UNIVAP / Instituto de Pesquisa e Desenvolvimento - IP\&D - Laboratório de Física e Astronomia, Brasil. E-mail: gimeneshumberto@outlook.com.

${ }^{2}$ Doutoranda em Física e Astronomia - Universidade do Vale do Paraíba - UNIVAP / Instituto de Pesquisa e

Desenvolvimento - IP\&D - Laboratório de Física e Astronomia, Brasil. E-mail: zuleika.sjc@bol.com.br.

${ }^{3}$ Doutor em Astrofísica. Docente e Pesquisador da Universidade do Vale do Paraíba - UNIVAP / Instituto de

Pesquisa e Desenvolvimento - IP\&D - Laboratório de Física e Astronomia, Brasil. E-mail: guga@univap.br.
} 


\section{INTRODUÇÃO}

A análise de séries temporais compreende dois tipos característicos de sinais: estacionário e não-estacionário, que se devem à natureza do fenômeno físico. Como o próprio nome já ilustra, sinais estacionários mantêm suas propriedades inalteradas ao longo de todo o tempo, não sendo necessário determinar onde as componentes de frequência ocorrem, apenas quais são elas. Sinais desse tipo são completamente caracterizados usando a Transformada de Fourier que os decompõe em suas componentes de frequência, demonstrando a contribuição de cada uma delas na composição do sinal, porém não indica a ocorrência no tempo.

Os sinais não-estacionários são mais complexos, pois representam fenômenos intermitentes, cujas frequências variam ao longo do tempo. Dessa maneira o interesse é determinar a ocorrência das componentes de frequência ao longo do tempo, sendo a Transformada Wavelet um método matemático intrínseco a esse tipo de análise.

A Transformada Wavelet é uma transformada linear que correlaciona um sinal com uma família de formas de onda que são bem concentradas tanto em tempo como em frequência (MALLAT, 2008). Tais formas de onda são denominadas time-frequency atoms, pois são as estruturas básicas na análise de qualquer sinal. Seja $f(t)$ uma função no tempo e $\left\{\varphi_{\_} \zeta\right\}$ uma família de formas de onda indexadas por $\xi$, sua transformada linear é definida como:

$$
T f(\xi)=\int_{-\infty}^{\infty} f(t) \varphi_{\xi}^{*}(t) d t=\left\langle f, \varphi_{\xi}\right\rangle
$$

A transformada wavelet pode ser definida em termos de (1), porém antes de entrar em detalhes, é necessário avaliar como suas formas de onda são definidas e as suas propriedades. Além disso, existem dois tipos de transformada, a contínua e a discreta, sendo que, no presente artigo, apenas a primeira será considerada.

Para que uma função $\psi(t) \in L^{2}(\mathbb{R})$ seja uma wavelet, é necessário que ela apresente algumas propriedades (SOMAN; RAMACHANDRAN; RESMI, 2010), como média zero (2):

$$
\int_{-\infty}^{\infty} \psi(t) d t=0
$$

E energia unitária (3):

$$
\int_{-\infty}^{\infty}|\psi(t)|^{2} d t=1
$$

Respeitando (2) e (3), é possível definir uma wavelet-filha pela dilatação $\boldsymbol{a}>\mathbf{0}$ e translação $b$ de uma wavelet-mãe $\boldsymbol{\psi}$ : 


$$
\varphi_{\xi}(t)=\psi_{a, b}(t)=\frac{1}{\sqrt{a}} \psi\left(\frac{t-b}{a}\right)
$$

Logo, substituindo (4) em (1), torna-se evidente que a transformada wavelet contínua de $f \in L^{2}(\mathbb{R})$ no tempo $\boldsymbol{b}$ e escala $\boldsymbol{a}>\boldsymbol{o}$ é:

$$
W f(a, b)=\int_{-\infty}^{\infty} f(t) \frac{1}{\sqrt{a}} \psi^{*}\left(\frac{t-b}{a}\right) d t
$$

A forma de onda escolhida para a análise das séries temporais foi a Morlet, dada pela equação (6):

$$
\psi(t)=\pi^{-1 / 4} e^{-i \omega_{0} t} e^{-t^{2} / 2},
$$

sendo seu gráfico representado na Figura 1:

Figura 1 - Função wavelet Morlet (Parte Real).

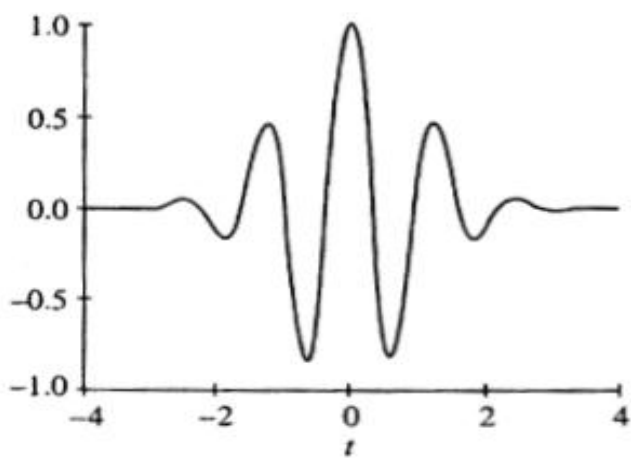

Fonte: Soman, Ramachandran e Resmi (2010, p. 33)

De forma intuitiva, a transformada wavelet funciona como um microscópio matemático em buscas de padrões, sendo que a wavelet-mãe é escolhida de acordo com as informações de interesse que serão extraídas. Basicamente, ela percorre todo o sinal, concebendo coeficientes de correlação que indicam o quanto o sinal se parece com as versões dilatadas e transladadas da wavelet-mãe, permitindo estabelecer uma conexão entre a frequência do sinal naquela região a frequência da respectiva wavelet. Dessa maneira, é possível conceber um espectro indicando as regiões de maior correspondência (Figura 2). 
Figura 2 - Ilustração do funcionamento da transformada Wavelet ao percorrer um sinal concebendo seus coeficientes.
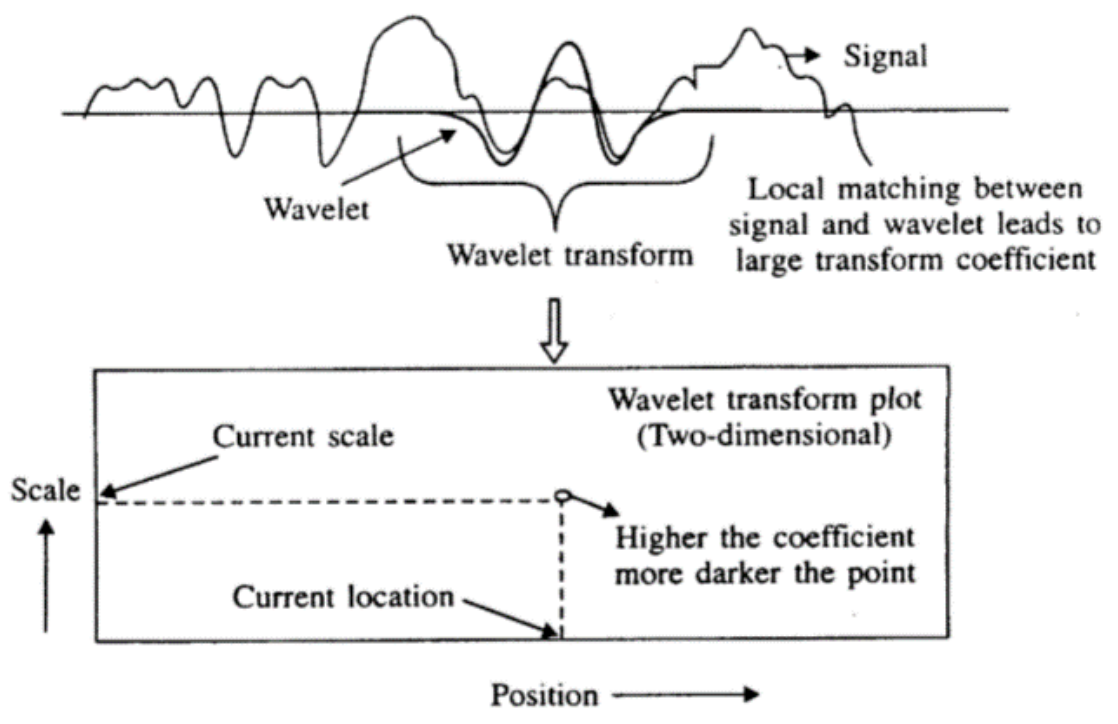

Fonte: Soman, Ramachandran e Resmi (2010, p. 34).

Uma vez que os coeficientes são determinados, uma representação tempofrequência é concebida, permitindo-se determinar quais componentes de frequência estão presentes e sua ocorrência no tempo, como na Figura 3.

Figura 3 - Representação bidimensional tempo-frequência da transforma wavelet.

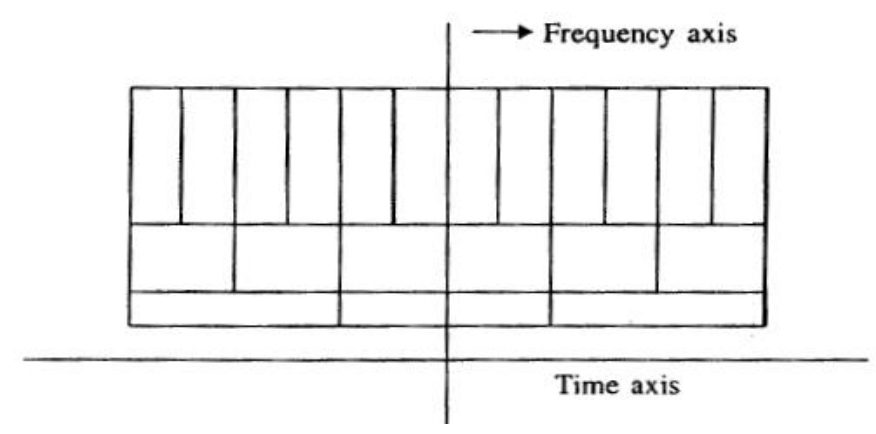

Fonte: Soman, Ramachandran e Resmi (2010, p. 45).

Porém, devido à incerteza de Heisenberg, é impossível obter uma boa resolução tanto em tempo como em frequência, sendo esse um dos problemas associados a esse tipo de transformada.

Assim, para compreender a Figura 3, é necessário ilustrar o que ocorre com a wavelet ao variar seu parâmetro de escala. Quando o coeficiente de escala é grande, a wavelet é dilatada, ao passo que, quando pequeno, ela é comprimida, como mostra a Figura 4. 
Figura 4 - Variação dos parâmetros de escala e translação.

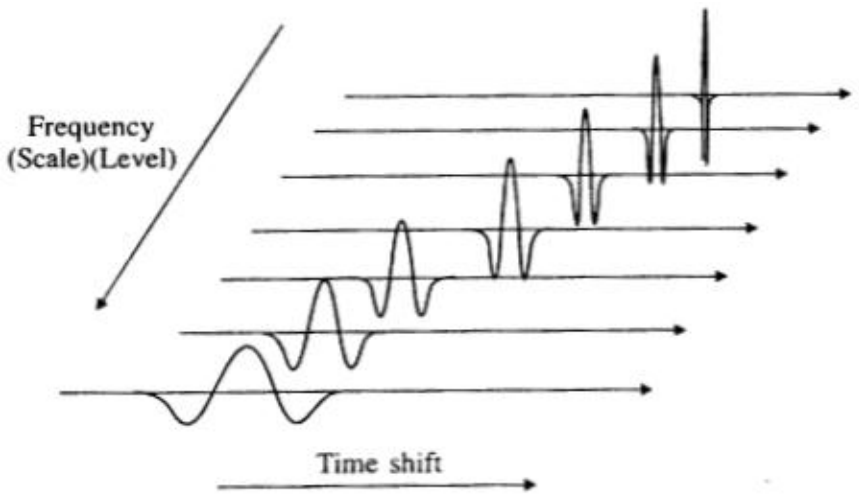

Fonte: Soman, Ramachandran e Resmi (2010, p. 33).

Uma wavelet dilatada apresenta grande suporte no tempo, sendo que essa propriedade é essencial ao se determinar baixas frequências, porém sua resolução temporal é ruim. Em altas frequências, a wavelet se comprime, aumentando sua resolução temporal.

Portanto, a transformada wavelet se apresenta como uma grande método matemático para análise de sinais não estacionários, como ocorre em rádio-emissões, sendo que o principal objetivo do presente projeto é a avaliação da eficiência da transformada em séries temporais associadas a eventos solares.

\section{METODOLOGIA}

Para a implementação da transformada wavelet, utilizou-se do ambiente computacional MATLAB, no qual se desenvolveu uma rotina para análise de séries temporais com base no algoritmo desenvolvido por Torrence e Compo (1998). Para avaliação de sua eficiência, utilizou-se de séries temporais associadas a rádio emissões solares tipo I e tipo III, registradas pelo espectrógrafo BLEN7M da rede eCALLISTO (BENZ et al., 2005; BENZ et al., 2009).

Detalhes da fenomenologia e análise das emissões tipo I registradas pelos espectrógrafos da rede e-CALLISTO são apresentados por (SODRÉ et al. 2011; SODRÉ; SILVA; FERNANDES 2012, SODRÉ; FERNANDES, 2013a; SODRÉ; FERNANDES , 2013b; SODRÉ; CUNHA-SILVA; FERNANDES 2015). Para as emissões tipo III, ver, por exemplo, MELÉNDEZ et al., 1999; SAWANT et al., 2002; FERNANDES et al., 2004; FERNANDES et al., 2008.

Cada evento é registrado em um arquivo FITS, que é composto por uma matriz em que as linhas representam um canal de frequência e as colunas as amostragens no tempo. Além disso, o arquivo contém informações técnicas, como o equipamento utilizado, altitude do local, tempo de registro, período de amostragem, entre outras. 
Os parâmetros necessários consistem do arquivo FITS e do canal de frequência, uma vez definidos, a transformada é aplicada sobre a série temporal definida pelo canal de frequência. Para avaliação do funcionamento do software, dois arquivos foram utilizados, caracterizando eventos solares distintos. O primeiro arquivo consiste no registro de grupos de emissões tipo III, registrados no dia 09/05/2015, das 16:29:38 UT às 16:45:00 UT, cujo espectro dinâmico é mostrado na Figura 5.

\section{Figura 5 - Espectro dinâmico de grupos de emissões tipo III registrados pelo BLENSW no dia 09/05/2015 das 16:30:00 UT às 16:45:00 UT.}

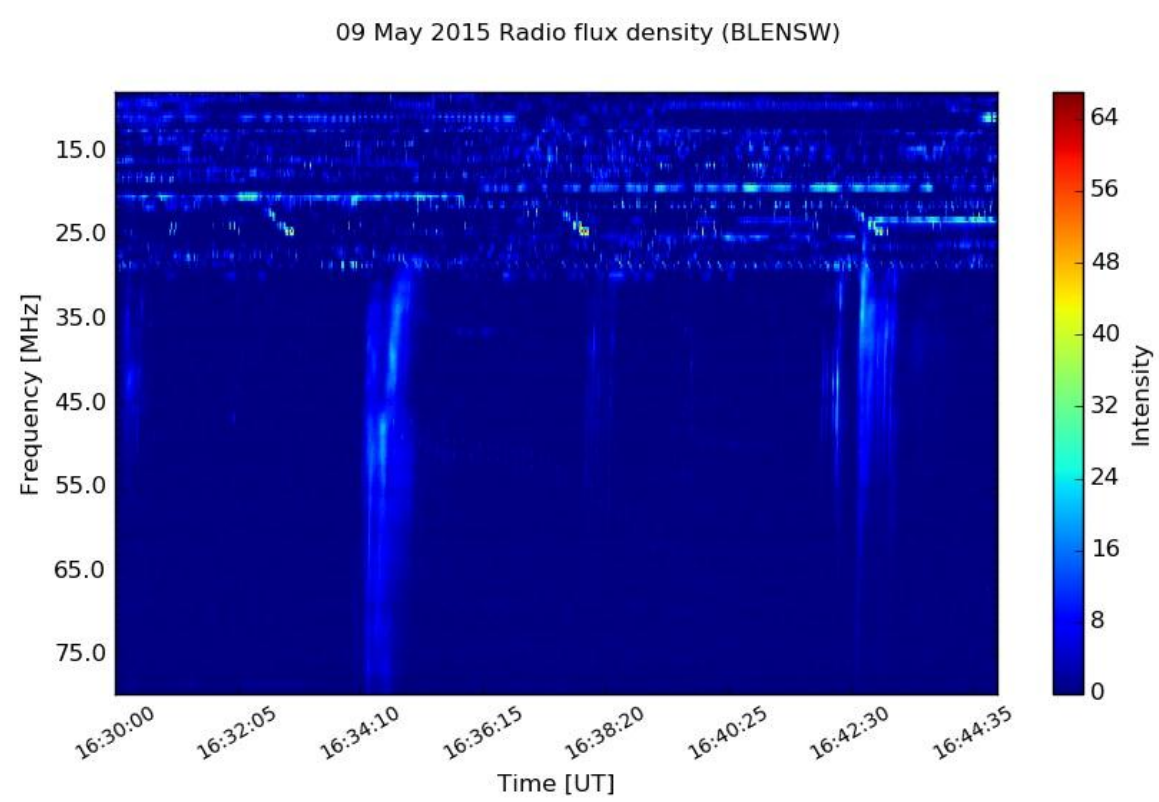

Fonte: Autor.

Para efeitos de análise, os canais de frequência 120 e 130, compreendidos entre $30 \mathrm{MHz}$ e $40 \mathrm{MHz}$, foram escolhidos, motivados pelas informações contidas no espectro, uma vez que se espera certa periodicidade nas regiões de maior amplitude que estão bem concentradas, como indicado na Figura 5. Dessa forma, espera-se que os coeficientes wavelet de maior relevância estejam presentes nessas regiões.

O segundo arquivo contém o espectro de uma sequência de emissões tipo I (caracterizando uma tempestade de ruído), registrada no dia 02/08/2010, das 11:00:31 UT às 11:15:31 UT (Figura 6).

Nesse caso, os canais de frequência 177 e 190, correspondendo às frequências entre $270 \mathrm{MHz}$ e $320 \mathrm{MHz}$, respectivamente, foram escolhidos, pois compreendem a região de maior atividade do fenômeno de acordo com o espectro. As saídas do programa para esses quatro canais, tanto do primeiro quanto do segundo, serão apresentadas na próxima seção. 
Figura 6 - Espectro dinâmico de uma tempestade de ruído composta por sequências emissões tipo I registrada pelo BLEN7M no dia 02/08/2010 das 11:00:31 UT às 11:15:31 UT.

02 Aug 2010 Radio flux density (BLEN7M)

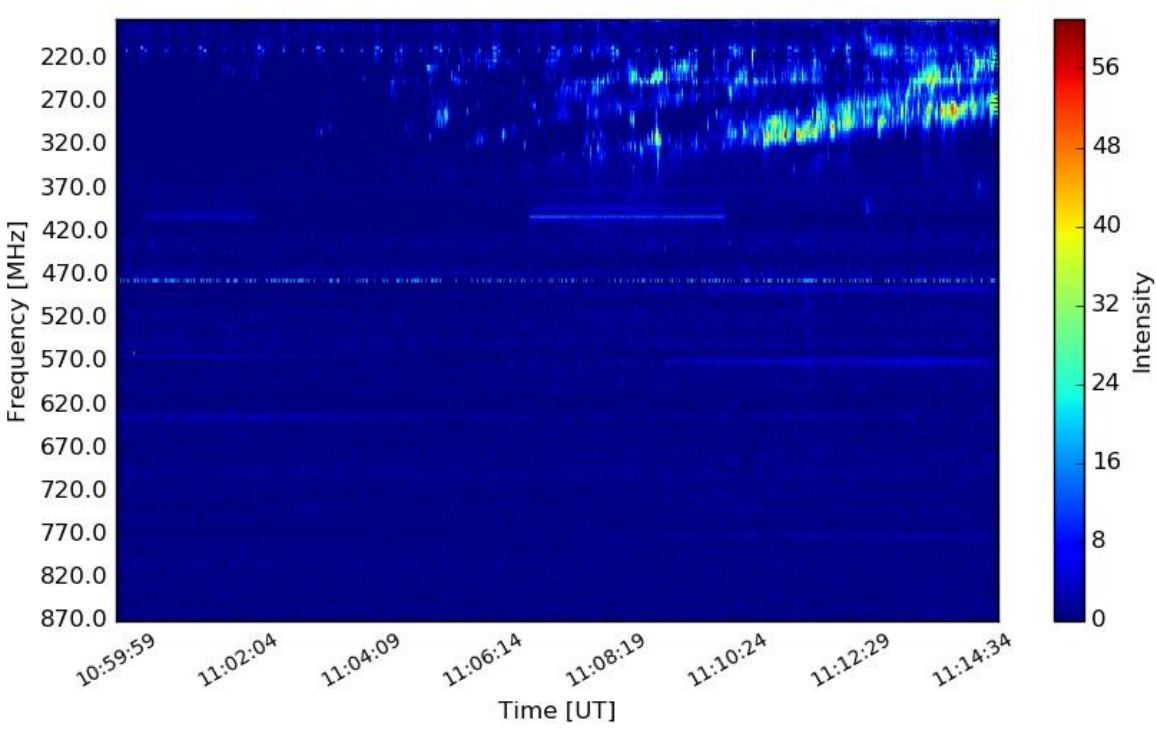

Fonte: Autor.

\section{RESULTADOS E DISCUSSÃO}

A discussão dos resultados obtidos será dividida em duas partes, de acordo com os eventos analisados: inicialmente os resultados preliminares das emissões tipo III e finalizando com as emissões tipo $I$.

A Figura 7 representa o espectro wavelet para a série temporal associada a emissões solares tipo III, no canal de frequência 120. Nota-se que as regiões que apresentam os maiores coeficientes apresentam um período em torno de 64 segundos, entre 16:33:36 UT e 16:36:28 UT. 
Figura 7: Periodograma da série temporal associada ao canal de frequência 120 da explosão do tipo III.

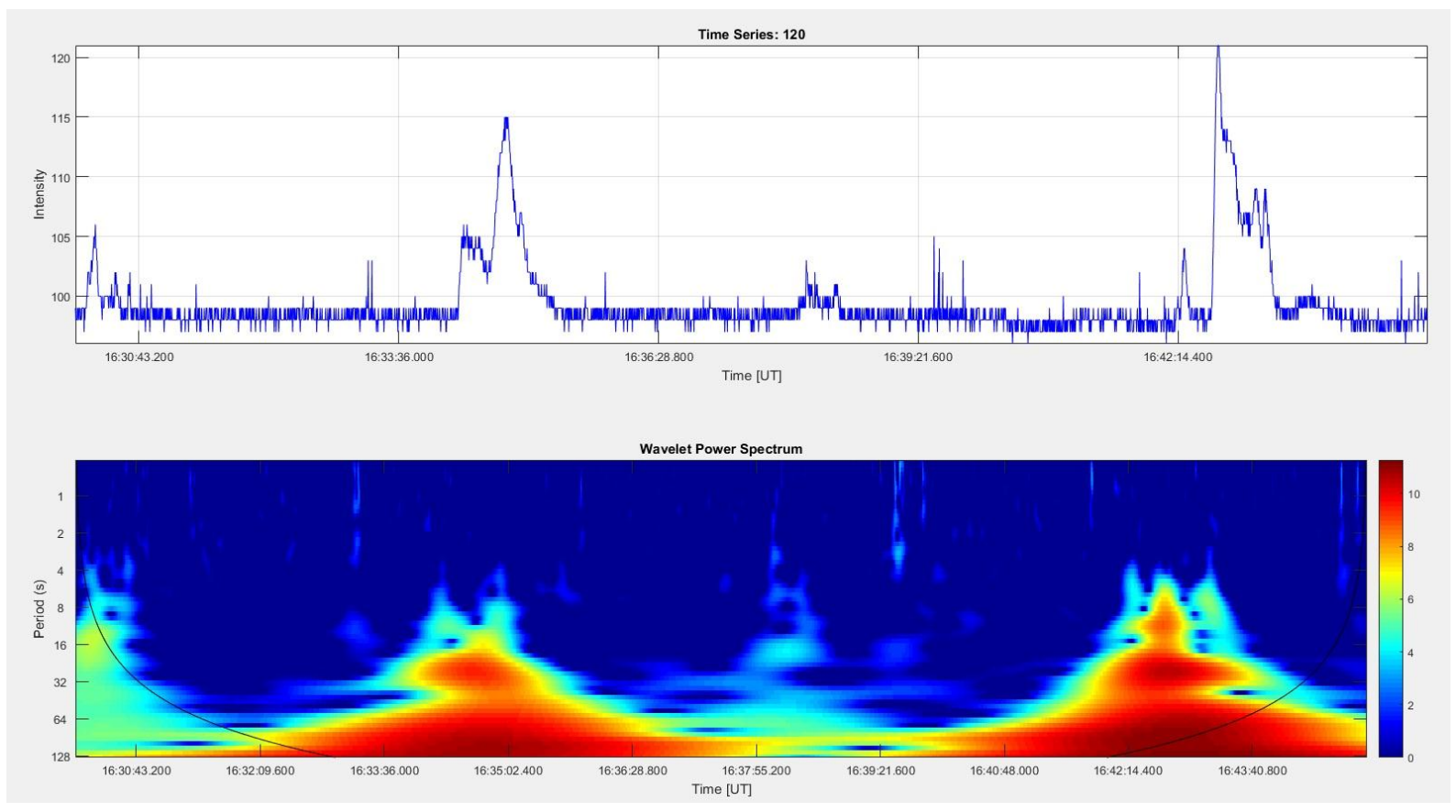

Fonte: Autor.

No caso do canal de frequência 130, a saída apresenta-se de forma similar ao canal 120, uma vez que representa uma região do espectro de comportamento semelhante, no que diz respeito às amplitudes. É possível observar que o período, no periodograma ilustrado na Figura 8, também está em torno de 64 segundos, entre 16:33:36 UT e 16:36:26 UT, apresentando uma mudança para um período de 32 segundos, quando se aproxima de 16:42:14 UT. 
Figura 8: Periodograma da série temporal associada ao canal de frequência 130 para o grupo de emissões tipo III.
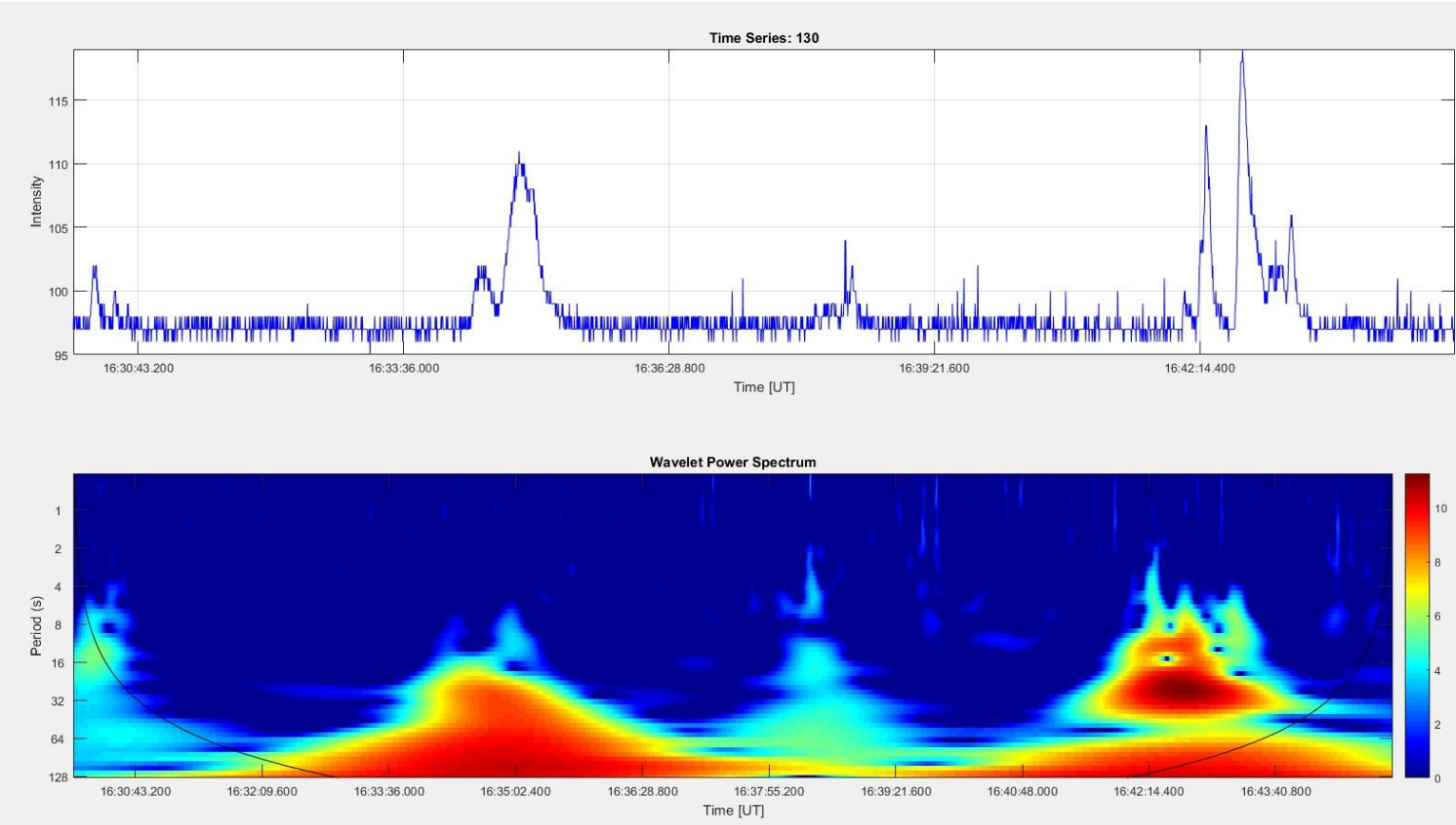

Fonte: Autor.

Para o caso das emissões tipo I, os canais de frequência analisados foram o 177 e 190, respectivamente. A Figura 9 representa a saída para o canal 177, apresentando um período quase constante de 64 segundos, entre 11:05:16 UT e 11:11:02 UT.

Figura 9: Periodograma da série temporal associada ao canal de frequência 177, da explosão do tipo I.

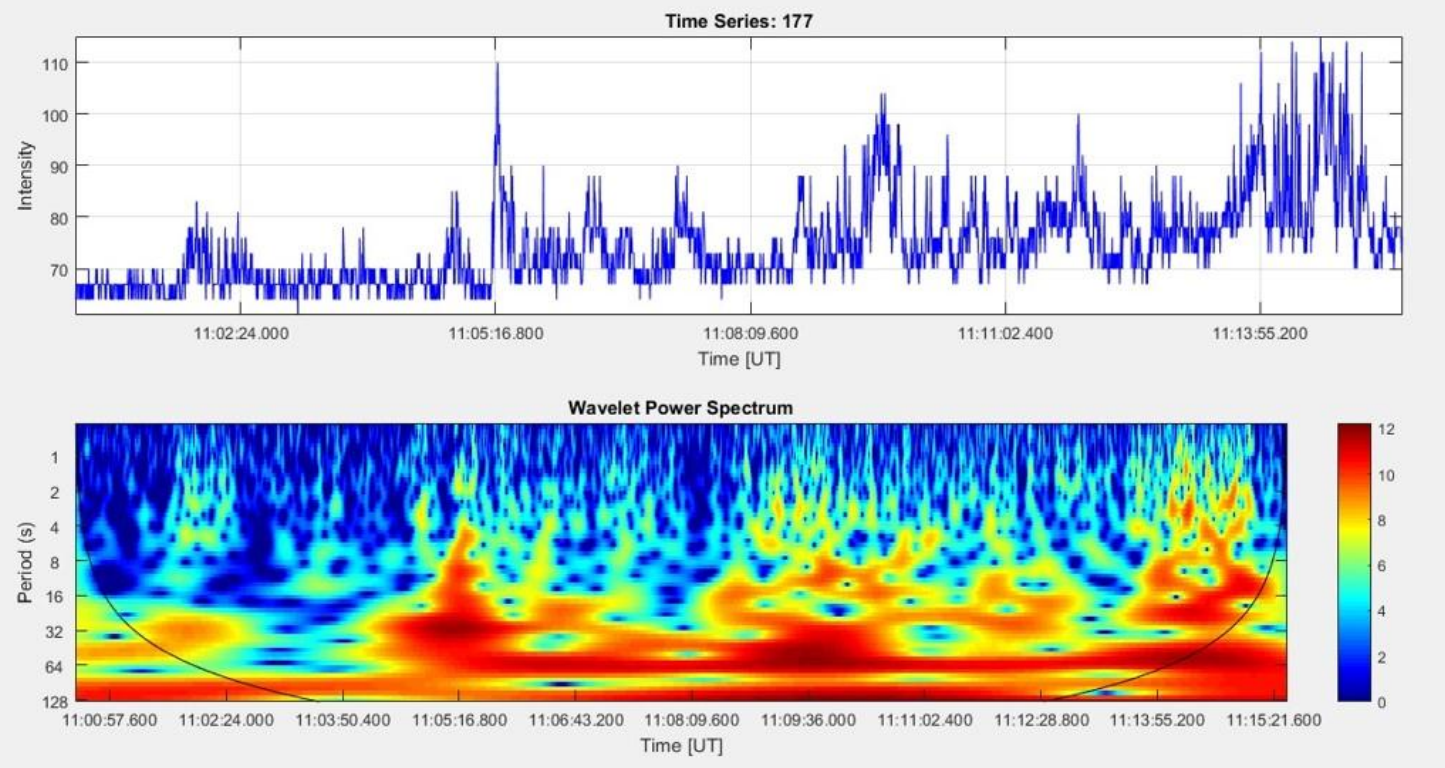

Fonte: Autor.

O periodograma do canal de frequência 190, ilustrado na Figura 10, por sua vez, 
é bem diferente do 177 , pois apresenta um período de 16 segundos, compreendendo, praticamente, toda a série temporal.

Figura 10: Periodograma da série temporal associada ao canal de frequência 190, da sequência de emissões tipo $I$.

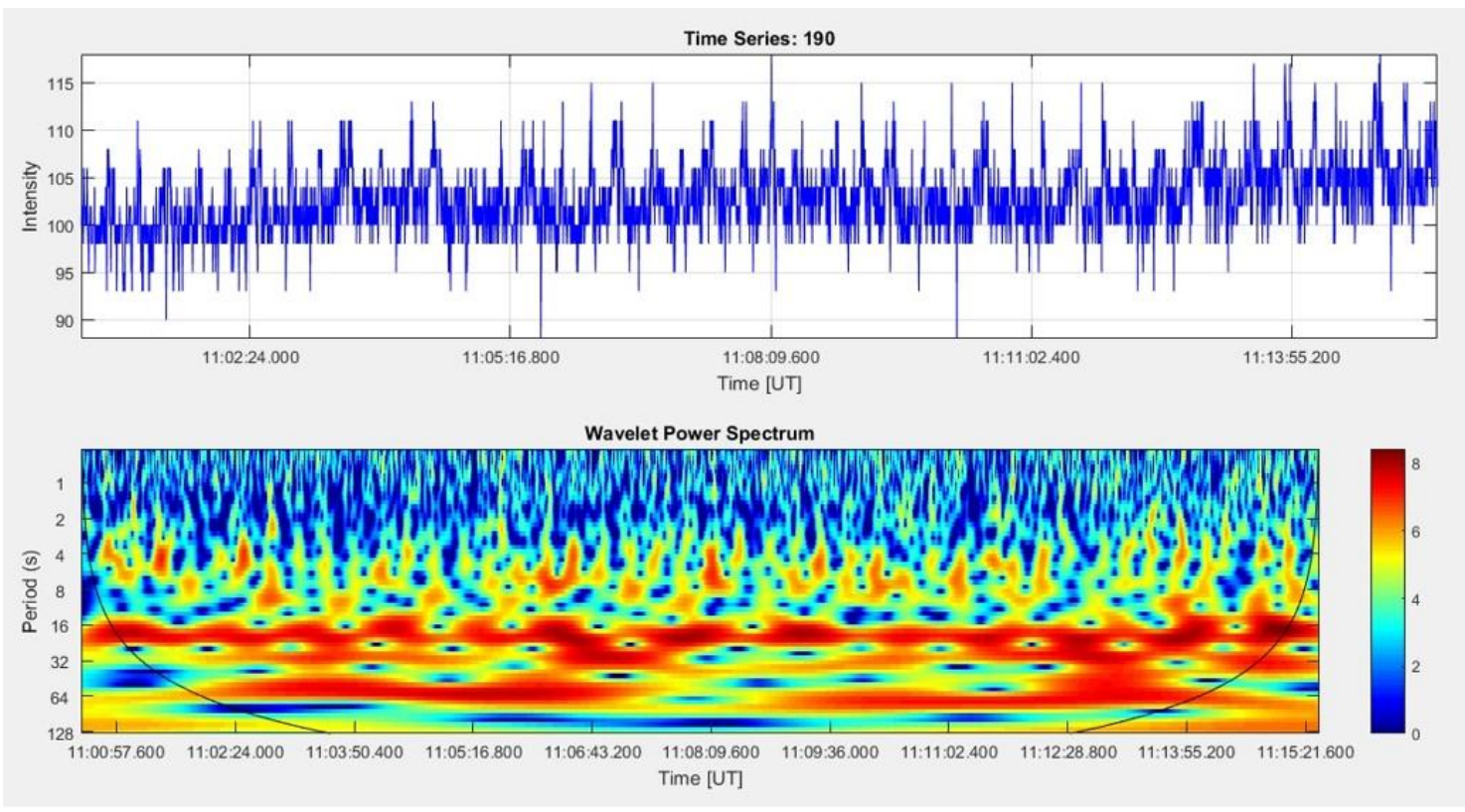

Fonte: Autor.

\section{CONCLUSÃO}

O presente trabalho apresenta resultados preliminares da aplicação de técnicas de wavelet, desenvolvidas em ambiente MATLAB, para a identificação de periodicidades em rádio emissões solares. Para testar a rotina desenvolvida, foram realizados testes de aplicação em dados de emissões solares tipo I (emissões intermitentes de banda estreita que ocorrem em longa duração, constituindo tempestades de ruído) e grupos emissões tipo III, registradas na faixa de frequências métricas (30 a $400 \mathrm{MHz}$ ) por espectrógrafos da rede e-CALLISTO.

Os resultados preliminares da aplicação, sumarizados nos periodogramas gerados e apresentados, apontaram períodos de 32 e 64 segundos para as emissões tipo III e de 16 e 64 segundos para as emissões tipo I.

Dessa forma, conclui-se que a aplicação de técnicas wavelet apresentou-se eficiente para a caracterização de periodicidades das emissões analisadas.

As investigações das características de rádio emissões nos domínios espectrotemporal (cadência temporal, intermitência, periodicidades ou padrões harmônicos por meio de análise wavelet, serão ampliadas para outros conjuntos de dados e os 
resultados, certamente, devem contribuir para a investigação dos mecanismos de emissão, uma vez que permitem inferir sobre a natureza fragmentada e intermitente dos processos de liberação da energia que geram essas emissões solares.

\section{REFERÊNCIAS}

BENZ, A. O. et al. A New Concept for Solar Radio Spectrometers, Sol. Phys., v. 226, p. 143-151, 2005.

BENZ, A. O. et al. A World-Wide Net of Solar Radio Spectrometers: e-CALLISTO, Earth, Moon and Planets, v. 104, n. 1-4, p. 277- 285, 2009.

FERNANDES, F. C. R. et al. Decimetric Fine Structures as a Possible Signature of Chromospheric Evaporation. Braz. J. Phys., v.34, n.4B, p.1792-1796, 2004.

FERNANDES, F. C. R. ET AL. Decimetric Fine Structures as Signatures of Plasma Emission Mechanism and Magnetic Morphology of Flaring Loops, Montréal, 2008. In: COSPAR SCIENTIFIC ASSEMPLY, 37. Procedding...Montréal, 2008.

MALLAT S. A Wavelet Tour of Signal Processing. 3. ed. Academic Press 2008.

MELENDEZ, M. J. L. et al. Statistical Analysis of High Frequency Decimetric Type III Bursts. Solar Phys, v.187, p.77- 78, 1999.

SAWANT, $H$. S. et al. Observation of harmonically related solar radio zebra patterns in the 1-4 GHz frequency range. A\&A, v.396, p.1015-1018, 2002.

SODRÉ, Z. A. L. et al.. XXII MPG - Mostra de Pós-Graduação, UNITAU, Taubaté SP, 2011.

SODRÉ, Z. A. L.; SILVA, R. D. C.; FERNANDES, F. C. R. VII Encontro de Verão de Física do ITA, ITA, São José dos Campos - SP, 2012.

SODRÉ, Z. A. L.; FERNANDES, F. C. R. Anais de Trabalhos Completos do XII EPG I XVII INIC, 2013a.

SODRÉ, Z. A. L.; FERNANDES, F. C. R. Revista Univap - Edição Especial - SimFAST, v. 19, p. $76-81,2013 b$.

SODRÉ, Z. A. L.; CUNHA-SILVA, R. D.; FERNANDES, F. C. R. Solar Phys., v. 290, p. 159-168, 2015.

SOMAN P. K.; RAMACHANDRAN I. K.; RESMI G. N.; Insight into Wavelets from Theory to Practice. 3. ed. Prentice-Hall of India: New Delhi. 2010. 305 p.

TORRENCE, C., COMPO, G.P. A Practical Guide to Wavelet Analysis. Bulletin of the American Meteorological Society, v. 79, n. 1, p. 61-78, 1998. 